Holland only require 11.5 per cent. of solids, of which 2.5 per cent. must be fat. Ohio's requirement is the same as New York's. Apparently it is a little more fair than the 13 per cent. of Massachusetts. Evidence of this is seen in the fact that in the latter State of 3549 samples examined in 1894, 1597 did not contain 13 but did contain 12 per cent. The enforcement of the law in Ohio is entrusted to the Dairy and Food Commissioner, and last year he was allowed over $\$ 50,000$ to expend. As a result he is able to report: "greater attention paid by dealers to the quality of of goods sold by them than has ever been done before. Immense amounts of bad goods have been shipped out of the State and manufacturers from all parts of the country are anxious to ascertain the requirements of law previous to sending goods into the State. In many cases manufacturers have informed me that they find it is necessary to put up one class of goods for Ohio and cheaper goods to be sent into States where laws were not enforced." He further declares "more work has been done in the prevention of fraud in the sale of food and drug products, and in the preservation of the public health thereby in the State of Ohio during the past ten years than in all the other States of the Union combined." All honor to Ohio! But shame on the other States!

But there are food laws which are manifestly unfair. Like many portions of our tariff laws they benefit only a few or a class.

About twenty years ago a French scientist named Mége-Mouriéz noticed that cows continued to give milk which contained cream, even though they were cut off from all fat-producing foods. Manifestly some of their reserve supply of fat must be transferred to the milk. He began to experiment with these fats and found that by proper chemic and mechanical means he could produce a compound closely resembling chemically and macroscopically the familiar butter. Physiologists tested it and found that for all practical purposes oleomargarine was as useful a food as butter. As it could be made for about one quarter the cost of butter, the discovery was hailed as a great boon for the poor. Suddenly the farmer awoke to the sitation. He was losing the market for his butter. Legislatures were appealed to. A very large proportion of the legislators were farmers themselves. Countless stories, most of them false, were concocted telling of the foul constituents and dreadful methods used in its manufacture. The result is a series of laws which are practically prohibitory and the loss to mankind of a valuable food. As physicians we should do our utmost to promote a reasonable regulation of the manufacture of such foods; for we know how sadly they are missed in the dietary of the poor.

Another article of food which was found to be frequently adulterated was cheese. The milk fats were left out by skimming the milk, and other fats, lard most commonly, worked in. In the detection of these frauds the microscope has been very valuable. Numerous laws for the protection of the public have been passed in the differeut States, which in some respects resemble the oleomargarine laws. The fight has not been as bitter, however, nor the laws as unfair. Thus in New York, Wisconsin and Minnesota, makers of "full cream cheese" are allowed to use an official stamp. To use this stamp on any cheese not "full cream" is to render one self liable to severe penalties. Ohio and Colorado go a step further and require the "skimmed" and "imitation" or "filled" cheeses to be so stamped with an official stamp several inches square. The other States have not troubled themselves very much in this matter.

Flour is only rarely adulterated, for as we noted at the outset, adulteration is practiced only to make money. Sugar is another great staple food which is now only rarely adulterated. Owing to the ease with which the taste and color could be imitated, vinegar has been much manufactured. Some States, Massachusetts for instance, have therefore established by law the percentage of apple substance which vinegar must contain as well as the per cent. of acetic acid, viz., $4 \frac{1}{2}$ per cent. Honey and maple syrup are two common articles of food which need constant inspection; and there are a multitude of others. Let me quote a few of those frauds detected by the Ohio inspectors in 1896 and punished by Ohio's laws; candy containing the poison anilin black; tomato catsup containing salicylic acid; canned peas colored by oxid of copper, oxid of lead and oxid of tin; black pepper containing buckwheat, roasted cocoanut shells and cayenne pepper; molasses adulterated with commercial glucose; buckwheat flour adulterated with wheat flour; cloves deprived of oil; green peas colored with the salts of copper; cider containing salicylic acid; raspberry jelly made of ether, alcohol and anilin red; current jelly composed of glucose syrup and salicylic acid; coffee adulterated with chicory, peas, corn and wheat bran. Truly the American is ingenious!

These facts are not secrets. Why, then, are there not more States with comprehensive laws? Why do not the States with good laws allow sufficient money to its agents to enable them to do good work?

More than seven billions of our money are invested annually in our food and drug product. Millions on millions of this finds its way to the dealer in adulterated products. And in these days it is the money power which secures legislation. Not always, however, and not necessarily. As physicians with a wide influence, let us agitate the matter, stir up and instruct public opinion, and it may not be many years before great results shall be attained both for the health and wealth of our people.

Reference Haudbook of Medical Sciences, "Food and Drug Inspec"Legislation Concerning Adulteration of Food and Drugs," Henry A. Riley.

Reports of Massachusetts Board of Health, 1879-1896

Report of Ohio Dairy and Food Commissioner, 1895 and 1896; "Food Character of Food Adulterations," Alexander J. Wedderburn.

\section{DIET IN THE CHRONIC CATARRHS OF THE} GASTRO-INTESTINAL TRACT.

Presented to the Section on Physiology and Dietetics, at the Forty-eighth Annual Meeting of the American Medical Association held at Philadelphia, June 1-4, 1897.

BY BOARDMAN REED, M,D.

PHILADELPHIA, PA.

In treating of diet in the chronic catarrhal inflammations of the stomach and intestines it is not intended to limit the discussion to those cases only which are usually classed as such. Our present exact methods show that chronic gastric catarrh in its earlier stages is rarely accompanied by nausea or vomiting and not always by pain in the stomach itself; also that cases of gastritis grave enough to have seriously impaired the health may be characterized by an increase rather than by a diminution of the appetite.

Again the same methods prove the frequent coex- 
istence of catarrhal disease in the small intestines and persistent constipation or constipation alternating with diarrhea when the disease has become more advanced. Obstinate chronic diarrhea points usually to an aggravated catarrhal inflammation of the intestinal mucous membrane complicated in many cases with ulceration.

A proper regulation of the diet is important in most derangements of health-perhaps in all. In those involving the digestive system it is peculiarly important. The science of dietetics presupposes that the appetite is not a safe guide in all cases and experience abundantly confirms this view. In the matter of quantity alone there are numerous patients who habitually eat too much and many more who, in consequence usually of a faulty secretion of the digestive glands, eat too little. It can not be too strongly emphasized, however, that patients suffering with chronic catarrhal inflammation of the digestive tube require a strongly nourishing diet. Their nutrition is apt to be low and commonly shows a strong tendency to sink lower. A portion of their food fails of its intended purpose by undergoing fermentation or putrefaction instead of digestion and assimilation. In this way there occurs not only a positive loss to the organism of much needed pabulum, but a still greater injury through the absorption of toxic substances resulting from these morbid processes.

To offset the depressing effects of this two-fold injury there is often a craving for food in abnormally large quantities, which when taken in the three usual meals daily, embarrass the system and aggravate the existing disease by overburdening the viscera, producing stagnation and retarding digestion with dilatation as an ultimate consequence. One remedy in such cases is to prescribe more highly concentrated and nourishing kinds of aliment in the most easily digestible forms possible, and when this does not suffice to fulfil the needs of nutrition without overtaxing the organs, then to prescribe smaller meals and more of them.

Numerous theories are current as to the particular forms of diet best adapted to these catarrhal affections and much diversity of practice has resulted. A reliance chiefly upon the cereals, vegetables and fruits has been urged, especially in France, by DujardinBeaumetz and others, and still has earnest and honest champions in some of our American sanitariums. This vegetarian system, in addition to its cheapness, has a number of manifest advantages in its comparative asepsis, its laxative tendency, its palatability for most patients and its valuable nutritive qualities, maintaining the strength well and when it agrees often putting on flesh rapidly. Yet the observations of many physicians and my own experience in its use indicate that it is not a safe diet in the diseases now under consideration, except possibly in the mildest forms of intestinal catarrh associated with constipation. These foods disagree because they are highly fermentable and often irritating to the chronically inflamed mucous membranes.

A nearly exclusive milk diet has its votaries and in certain cases proves curative, especially when the milk can be obtained pure and comparatively fresh from the cow. The ordinary commercial milk of cities is always from one to three days old when served to the customer, and is often handled in such a careless manner as to become dirty and infected with all manner of germs, even when it escapes the grosser impurities resulting from its admixture with contaminated water and adulteration of drugs to counteract the effects of too long keeping. Happily, however, this difficulty has recently been well overcome in many of the larger cities and it is now possible, for the well-to-do classes at least, to obtain, in sealed jars, milk which when furnished by honest dealers, is comparatively pure and aseptic, and usually not more than twelve to twenty-four hours old.' Such a milk properly modified or diluted may be given very largely in many catarrhal cases with the utmost advantage, especially when a little good bread or toast or sometimes a few eggs are added to the daily allowance. But this method employed in a merely routine or empirical way without any exact knowledge as to either the motor power of the stomach or the faults in its secretory function is capable of doing harm. When there is much gastric dilatation or even marked atony, milk or any liquid taken in large quantities is liable to aggravate; and when the stomach is badly infected the same food is apt to ferment rapidly. It suits particularly well as a rule, in those forms of chronic diarrhea where the pancreatic secretion has been well preserved. However, as Ewald so well says: "It must also not be forgotten that an exclusive milk diet is a kind of slow starvation, and that to live on milk alone would require much larger quantities than the capacity of the stomach would allow." ${ }_{2}$ Hence such a diet can not be long persevered in without unduly lowering the strength.

In chronic gastric catarrh Ewald and most of the German authorities advise a mixed diet without any very severe restrictions. They allow meats, eggs, milk and many of the carbohydrates, proscribing, however, freshly baked breads, etc., as well as the coarser vegetables, strong acids, sharp condiments and the stronger liquors. They recommend that the starches shall have been changed so far as possible into dextrin before their ingestion and object to dishes cooked with much fat. Ewald particularly objects also to fat meats and the oilier kinds of fish, hard boiled eggs and the tendinous parts of meat. Boas ${ }^{3}$ takes very similar ground, but like various other German writers, prescribes diet tables with definitely weighed proportions of the proteids, fats and carbohydrates for each meal. Both Ewald and Boas very properly caution against too sweeping and arbitrary prohibitions of whole classes of foods in the diet of dyspeptics generally, since every paticnt has idiosyncrasies which need to be studied individually.

The American method of limiting these patients for a time mainly to lean meat and hot water, though on a priori grounds irrational and unsafe, has borne well the crucial test of experience in a large number of cases in the hands of several able physicians. In the bad cases the patients are put at once on the pulp of broiled lean beef or beef which having been finely chopped and deprived of all the fat and tendinous parts is afterward broiled in small cakes. With these is given usually a very small portion of stale bread or toast without butter and a very little raw celery or lettuce-sometimes also asparagus tips or stewed celery with occasionally one orange or baked apple

\footnotetext{
Dr. Samuel G. Dixon, president of the Academy of Natural Sciences, Philadelphia, has called my attention to the fact that sometimes the men who deliver these jars refill them without cleansing, which would be highly dangerous when they have stood for hours in infected sick rooms. B. R.

The Diseases of the Stomach, by Dr. C. A. Ewald, New York, D. Appleton \& Co., 1894 Diagnostik u. Therapie d. Magenkrankheiten, von Dr. I. Boas. Part il,
Leipzig, George Thieme, 1895 .
} 
daily or a few white grapes, the skins and seeds being rejected. Coffee and tea may or not be allowed, but the patient is required by most advocates of the method to drink from one to two quarts, and sometimes three quarts, of hot water every day. As improvement sets in and fermentation lessens, the number of articles permitted is gradually enlarged, but upon the first sign of a relapse the strict meat diet is resumed.

In other cases where the digestion is somewhat better, lean meats are allowed from the start in the usual forms of tender beefsteak and lamb chops, the fat and gristle being rejected.

Carried out with extreme rigorousness in cases adapted to it, this system of diet will in the course of four or six weeks, even without the aid of stomach washing, often greatly lessen fermentation. It will ameliorate markedly many stubborn cases of gastric or intestinal catarrh and according to the testimony of some of its most zealous champions, may even, when followed up later in a modified form, effect a radical cure, though not usually before the end of two or three years.

The more enthusiastic advocates of this exclusive diet recommend it as a rule in all cases of gastrointestinal catarrh, and it can not be denied that in a considerable proportion of such cases when intelligently directed and faithfully carried out, it does well. It supplies an abundance of highly nourishing albuminoid material in a form which is comparatively easy of digestion, and at the same time is very much less fermentable than most other foods. It is also of small bulk, except when inordinately large quantities of water are taken, thus aiding in overcoming dilatation of the stomach and bowels. The chief objection to it is that the proportion of carbohydrates and fats is far too low to support nutrition well for any great length of time. Then, too. the large quantities of hot water often required to be taken with the meat in order to keep up a sufficiently active elimination, have important contraindications. When the heart is very weak, a serious embarrassment may result from throwing into the circulation such unusual amounts of fluid, and when there is much dilatation or even marked atony of the stomach walls, these conditions are almost sure to be aggravated by taking in so much fluid.

Another objection to the routine employment of such an almost exclusive meat diet is that it is excessively stimulating to the gastric glands in cases where hyperchlorhydria complicates chronic gastric catarrh.

Until recently the possibility of the coexistence of these two conditions was not recognized, but various observers have shown of late that we may have an acid gastritis that is a true catarrhal inflammation, along with a highly excessive secretion of hydrochloric acid. My own clinical work has confirmed this view. Experiments conducted in my laboratory have demon. strated, as mentioned in a previous paper, "that a meat diet powerfully excites the secretion of hydrochloric acid, thus tending to exaggerate the condition of hyperchlorhydria.

Numerous patients were put on a diet of meat mainly and the stomach contents analyzed every one to two weeks after the usual Ewald test breakfast. Some of these patients had in the beginning a normal

4 "The Excessive Secretion of Hydrochloric Acid by the Stomach and its possible serious Consequences." By Boardman Reed, M.D., Inter
national Clinics, vol. i, seventh series, 1897. proportion and others an excess of hydrochloric acid. The result was almost without exception a decided increase of the acid while on the diet. This was especially true in all the cases of the hyperchlorhydrics, except where the stimulating effect of the diet was counteracted by large doses of an alkali and some. times even in spite of this. Evidently, therefore, this diet however useful in suitable cases, would be unsafe as a routine method in all, without thorough analysis and tests in every case.

Personally, I have found more difficulty in getting patients to carry out the meat-and-hot-water method, with the necessary strictness, for many months together, than in inducing them to submit to lavage with a diet somewhat more liberal though on similar lines. My results, too, in chronic gastric catarrh have been better since I have been employing lavage along with a diet in which lean meat, eggs and milk predominate, excluding sugar, shellfish, hot or fresh breads, fried foods, all the less digestible vegetables as well as the coarse cereals and restricting starch foods to a few of the blandest in small amount. Stimulants and the stronger condiments are cut off as a rule, to which there are some exceptions. All the more acid fruits, especially those with small seeds, and all hard and gritty articles incapable of perfect solution, are strictly forbidden. In the worst cases I frequently, at first, prescribe either the pulp of lean beef or juice expressed from the same as almost the only food for a few days. When perfectly fresh eggs can be had they are usually added, being eaten either raw, soft boiled or poached. The whites beaten up and taken raw agree well even when the stomach is very irritable and make an ideal food. Then gradually the dietary is enlarged, watching the effects upon the urine, mucous secretion, body weight and condition generally.

In the pronounced cases of gastric catarrh with an excess of hydrochloric acid (acid gastritis) I often find it necessary to restrict the dark meats especially, ordering eggs and milk instead, with the gluten preparations and a small amount of white wheaten bread, preferably in the dryer forms so as to obtain a larger admixture of the saliva. The farinaceous food in these cases should be predigested so far as possible by heat. It is sometimes well to let the patient take daily one lunch or light meal consisting mainly of bread or other preparations from grains, changed so far as it is practicable by heat into dextrin, as in the form of toast or zwieback or the various malted foods. Good fresh milk is a good accompaniment of such a meal. Otherwise he should be advised to take whatever carbohydrates there may be allowed, in the early part of one or more meals, following these later with meat or eggs. In this way the salivary digestion is given an opportunity of proceeding well on toward its completion before the time - usually about twenty to thirty minutes from the beginning of the mealwhen the secretion of hydrochloric acid has become large enough to arrest the process.

In the cases of gastric atrophy in which there is practically no longer any secretion in the stomach, I have not found a meat diet to suit well as a rule, even when full doses of hydrochloric acid and pepsin are given. Sometimes eggs raw or lightly boiled agree better. Here, however, the best grades of the cereal preparations made of finely ground and bolted flours or meals with milk peptonized or not, according to the condition of the pancreatic and intestinal secre- 
tions and with the addition usually of a certain amount of eggs, has seemed to succeed best.

But in the cases of chronic gastric catarrh in which the secretion of the hydrochloric acid is merely diminished, the results from a diet of meat and eggs chiefly are often excellent. To supplement the work of the stomach glands hydrochloric acid can then be administered in quite small doses with usually the happiest results.

Observations in my laboratory have shown beyond question that 0.3 to 0.6 c.c. of the dilute hydrochloric acid given during the period of digestion in such cases tend strongly to promote the secretion of the gastric glands, so that it is not uncommon to find after a few weeks of such treatment, even when not conjoined with other roborant measures, a largely increased percentage of the acid as shown by analysis after the usual test meal.

In intestinal catarrh the selection of the diet must turn largely upon the condition of the stomach digestion. When the case is complicated by hyperchlorhydria the diet above recommended for that condition will generally be the most suitable. These are cases in which a milk diet frequently gives the best result. The same with the cautious addition of meat juice and eggs will usually agree well in cases of intestinal catarrh with very small or absent digestive power in the gastric glands, provided any deficiency in the secretion of the pancreatic juice shall be made up by the administration of a good active extract of pancreas.

When the intestinal catarrh is conjoined with good stomach digestion--a not very frequent combination however-there may be a full allowance of meat along with the blandest vegetables and farinaceous foods, though even here milk and eggs will often agree better.

\section{PHYSIOLOGY OF THE COLON, SIGMOID AND RECTUM.}

Presented to the Section on Physiology and Dietetics at the Fortyeighth Annual Meeting of the American Medical Association, at Philadelphia, Pa.. June 1-4, 1897.

BY J. G. CARPENTER, M.D.

STANFORD, KY.

In obscure cases of disease of the rectum and sigmoid flexure, inversion of the trunk seventy to eighty degrees, dilatation of the anus with Sims' speculum, inflation of the bowels and electric or reflected light (the former is preferable), are indispensable to a thorough ocular examination and diagnosis. In suoh cases I have used a rubber tube, eighteen inches long, with good results. By inversion of the trunk seventy or eighty degrees the pelvic and abdominal contents gravitate toward the diaphragm; a vacuum is formed in the rectum and sigmoid which become inflated with air under forced expiration and anus patulous and retracted with a Sims' speculum; the mucous folds are effaced, and the bowel has almost the appearance of a straight tube; on inspiration the bowel collapses and presents a curved tube, the mucous folds resuming their normal position.

The upper end of the rectum is much smaller than any other part of it, and presents a cavity closed from the sigmoid flexure, because the circular muscular

\footnotetext{
1 At Winchester, Ky., June, 1886, the author read a paper before the Kentucky State Medical Society on ulceration of the sigmoid cavity "Inversion of Trunk, Electric and Reflected Light in Diagnosis and Treatment,' which was published in the American Practitioner and News, Vol. 2, New Series, No. 17 , August 21, 1886, at Louisville,
On Nov. 30 , 1885, the writer was the first to do sigmoidoscopy.
}

fibers of the latter act as a sphincter, except during forced expiration and defecation; at such time the rectal cavity and lower part of the cavity of the sigmoid flexure seem one. Gray's "Anatomy," page 806, states that the sigmoid flexure is the narrowest part of the colon.

The rectum is cylindric, not sacculated like the rest of the large intestine. It is narrower at its upper part than the sigmoid flexure, gradually increasing in size as it descends and immediately above the anus presents considerable dilatation. It is capable of acquiring enormous size; when distended it is funnelshaped and naturally is a closed cavity caused by approximation of Houston's folds and action of the circular muscular fibers. "The sigmoid flexure has great mobility, with the hand introduced into the bowel a point above the umbilicus has been reached."

Ocular examination of the cavities of the rectum and sigmoid flexures, though one is expert in handling instruments and focusing reflecting light on the parts to be seen, is no easy feat to accomplish except by practice. The subject to be examined should be slender and emaciated and willing to bear some glight pain and discomfort in the accomplishment of inspection; the sphincter ani should be previously dilated or relaxed and dilatable. If sunlight is reflected into the rectum the head of the patient should be toward the sun, if other light be employed it should be to one side-the left is preferable. The trunk must be inclined to the left side and inverted to an angle of seventy or eighty degrees and the thighs flexed; both should be supported on hard pillows. The speculum (Sims') is introduced into the anus and rectum, retracting the former, the lower and about half of the middle third of the latter with the coccyx. The nates must also be retracted. The anterior rectal wall, or recto-vesical wall, and anterior wall of sigmoid flexure are to be pressed forward out of the way by the bluntpointed sound or applicator, or by inflation of air, the retraction of the anus and rectum pressing the anterior rectal wall and the anterior wall of the sigmoid flexure forward. The reflection of light or use of the electric lamp introduced within the bowel must be done simultaneously with forced and prolonged expiration.

The function of the sigmoid flexure is a receptacle for the feces as they pass from the descending colon, it being closed at its lower end by circular muscular fibers separating the sigmoid flexure from the rectum. The shape of the sigmoid flexure lessens or breaks the force of gravity of the feces; if the bowel at this point was a straight tube the intestinal contents would descend at once to the anus and cause continual inclination to defecate in the sitting or erect position. When the sigmoid flexure becomes filled normal reflex action by the spinal norves is produced, causing contraction of the circular and retraction of the longitudinal muscular fibers, by which the length and lumen of the bowel are made less above; the circular muscular fibers of the lower end of the sigmoid flexure and those of the rectum, the sphincters ani included, relax and with the volition of the patient defecation is accomplished, the lungs being inflated, and the diaphragm and abdominal muscles contracted, thus lengthening the contents of the abdominal cavity. Another function of the sigmoid and rectum and colon is to absorb nutritive enemata and sustain life in gastro-intestinal diseases. Physiologically the rectum is a closed and empty cavity (except during forced expi- 